\title{
Influencia cultural en la motivación para el aprendizaje del inglés como lengua extranjera*
}

\author{
Percepciones de los estudiantes de séptimo semestre de Pedagogía \\ en Inglés de la Universidad Distrital Francisco José de Caldas \\ (Colombia), y de la Universidad Mayor (Chile)
}

\section{Laura Beltrán Arias**}

Fecha de recepción: 20 de abril de 2015

Fecha de revisión: 17 de mayo de 2015

Fecha de aprobación: 2 de junio de 2015

\section{Resumen}

La influencia cultural es un aspecto determinante en el aprendizaje de una lengua extranjera. Esta investigación contrasta las percepciones de los estudiantes colombianos y chilenos de séptimo semestre de Pedagogía en Inglés en lo relacionado con la influencia que tienen algunos aspectos culturales en la motivación para el aprendizaje de una lengua extranjera. Es un estudio mixto llevado a cabo con 49 estudiantes de séptimo semestre, 26 de ellos provenientes de la Universidad Distrital Francisco José de Caldas (Bogotá, Colombia) y los 23 restantes de la Universidad Mayor (Santiago, Chile). Se logró reconocer por medio de encuestas semi-estructuradas y observaciones, que los participantes consideran que sus respectivas culturas no les motivan a aprender inglés

* Artículo de investigación científica financiado por el Centro de Investigaciones y Desarrollo Científico de la Universidad Distrital Francisco José de Caldas.

** Laura Beltrán Arias, estudiante de noveno semestre de Licenciatura en Educación Básica con Énfasis en Inglés, perteneciente al grupo de investigación Calidad y Saberes del Centro de Investigaciones y Desarrollo Científica de la Universidad Distrital Francisco José de Caldas. Dirección de correspondencia: Carrera 97 \# 40-A 21, sur, Bogotá. Correo electrónico: Ibeltrana@correo.udistrital. edu.co, I13260@gmail.com. 
como lengua extranjera, aunque reconocen la incidencia de este idioma como parte de la globalización, aspectos históricos, de desarrollo, etc.

\title{
Palabras clave
}

Cultura, motivación, aprendizaje de lengua extranjera, inglés como lengua extranjera, percepciones de los estudiantes.

\section{Cultural influence on motivations for learning english as a foreign language}

\begin{abstract}
Cultural influence is an important issue in foreign language learning. This piece of research contraststhe seventh semester of Bachelor of Arts in English Language students' from Universidad Distrital Francisco José de Caldas (Bogotá, Colombia) and Universidad Mayor (Santiago, Chile) perceptions about the cultural aspects which encourage them to learn it as a foreign language. It is a mixed study carried out with the participation of 49 seventh semester students, 26 of them from the Universidad Distrital and 23 students from the Universidad Mayor. It achieves to recognize through semi-structured surveys and observations that the participants consider that their respective cultures do not encourage them to learn English as a foreign language, even if they recognize the influence of this language as part of the globalization process, historical events, development, etc.
\end{abstract}

\section{Keywords}

Culture, motivation, learning of a L2, english as L2, students' perceptions. 


\section{Influência cultural na motivação para a aprendizagem do inglês como língua estrangeira}

\section{Resumo}

A influência cultural é um aspecto importante quanto à aprendizagem de uma língua estrangeira. Esta pesquisa contrasta as percepções dos estudantes do sétimo período de pedagogiaem inglês de da Universidad Distrital Francisco José de Caldas (Bogotá, Colômbia) e de da Universidad Mayor (Santiago, Chile) sobre os aspectos culturais que os motivam a aprender este idioma como língua estrangeira. É um estudo misto realizado com 49 estudantes de sétimo período, 26 deles provenientes da Universidad Distrital e os 23 restantes da Universidad Mayor. Foi possível reconhecer por meio de questionários semiestruturados e observações, que os participantes consideram que suas respectivas culturas não lhes motivam a aprender inglês como língua estrangeira, ainda que reconheçam a incidência deste idioma como parte da globalização, aspectos históricos, de desenvolvimento, etc.

\section{Palavras-Chave}

Cultura, motivação, aprendizagem de língua estrangeira, inglês como língua estrangeira, percepções dos estudantes. 


\section{Introducción}

El presente artículo es un análisis comparativo (colombo-chileno) pensado con base en el actual interés social por el aprendizaje del inglés dentro de estos dos contextos hispanoparlantes (aplicación de programas de bilingüismo); especialmente han sido investigadas las percepciones de algunos estudiantes de la Licenciatura en Inglés de la Universidad Distrital y de Pedagogía en Inglés de la Universidad Mayor, para determinar qué aspectos de su cultura les motivan a aprender inglés. Así, se busca identificar desde la mirada de los estudiantes cuáles aspectos de la cultura a la que pertenecen los han motivado para llevar un proceso de aprendizaje de esta lengua, y poder establecer cuáles de estos pueden ser fortalecidos con el propósito de mejorar los niveles de aprendizaje del inglés, que como lengua extranjera dentro del contexto colombiano y del contexto chileno está tomando gran fuerza.

Para lograr analizar de manera más concreta estos aspectos culturales se realizaron encuestas (validadas por el Semillero de Investigación al cual pertenece esta investigación) a estudiantes colombianos de la Licenciatura en Inglés de la Universidad Distrital, y los estudiantes de la Pedagogía en Inglés de la Universidad Mayor, con el objetivo de conocer cuáles son sus percepciones acerca de la influencia de la cultura sobre las motivaciones de los estudiantes para estudiar el inglés como lengua extranjera, lo que tangencialmente nos permite conocer algunas de las razones por las cuales los estudiantes eligieron la docencia como su profesión. Igualmente, es de tener presente la importancia del inglés como herramienta global para la interacción (lengua franca) (Universia, 2013) y el intercambio cultural dentro de los diferentes contextos y espacios que se le abren (centros educativos, actos culturales y artísticos, política, economía, etc.).

Los resultados obtenidos a partir de esta investigación dan cuenta que los estudiantes consideran su propia cultura poco motivante en cuanto al aprendizaje del inglés; por el contrario, en relación con sus intereses personales y los aspectos culturales de países angloparlantes, estos son más "interesantes, llamativos y motivantes", de acuerdo con lo expuesto en los instrumentos de investigación. Aborda teóricos como los de Patrick Moran (2001), quien trata a profundidad la cultura, sus dimensiones y su relación con la educación, de manera que permite el conocimiento y análisis de la influencia cultural en el aprendizaje. 


\section{Concepto de cultura}

Moran (2001), aborda la cultura en relación con la educación como:

El camino evolutivo de la vida de un grupo de personas, que consiste en un conjunto de prácticas asociadas a un conjunto de productos, con base en un conjunto de puntos de vista sobre el mundo compartidos entre los miembros del grupo, y que figuran dentro de contextos sociales específicos (p. 57).

Acorde con el concepto de cultura de Moran, Cavalli (2007) la plantea como una recolección de conocimientos y de creaciones traídas a la realidad por medio del lenguaje. Por lo que además establece que esta acumulación a nivel global es producto de contribuciones provenientes de diferentes individuos que han pasado de una generación a otra, y que son compartidas por diferentes grupos sociales de modo que influyen y transforman las vidas de los miembros de la misma de manera continua.

Otro concepto relevante puede observarse en Kramsch (1998, p. 59), que define la cultura en relación con el lenguaje y la entiende como un ambiente en una comunidad discursiva que comparte un espacio social y una historia común, así como los imaginarios forjados a través de las percepciones concebidas como grupo o comunidad. De modo que cualquier experiencia que un individuo pueda tener dentro de determinada cultura podrá afectar los múltiples aspectos de su identidad personal (género, edad, religión, clase, etc.) lo que además altera varias circunstancias de la vida del individuo.

Arabski (2011) plantea algunas ideas claves acerca de la cultura, como son:

Culture is elemental: People are exposed to and embedded in their own culture since birth so deeply that they may occasionally fail to recognize cultural foundations of their belief and value systems, attitudes and emotions. Their background in the frame of reference for contacts with other cultures and that is one of the reasons why reflection about this background is so important to make sense of other cultures (p. 24).

Donde es posible reconocer a la cultura como aspecto vital y natural en la vida de las personas de una comunidad y en relación con su manera de percibir a los demás. 
De esta manera el sujeto se encuentra permeado por la cultura desde el mismo momento de su nacimiento, hace parte de su diario vivir, su formación, el establecimiento de sus parámetros y calidad de vida, como de muchos otros aspectos que a través de su formación le van llevando a ser la persona que es (Levi, 2007, p. 107; Moorjani y Field, 1988). La cultura también puede ser evidenciada en un grupo específico en el que las personas que la conforman tienen la oportunidad de compartir valores, historias, modos o reglas de comportamiento, entre otras (Lindsay et al., 1999). Así existe la posibilidad de la creación de lazos que generan un reconocimiento del otro en tanto que es un sujeto participativo dentro de la comunidad y la cultura a la cual pertenece.

Shiarev y Levy, (2004) definen cultura como: "set of symbolicsystems, including knowledge, norms, values, beliefs, languages, art, customs, habits and skills learned by members of a given society" (p. 110). Esta definición implica que la cultura es aprendida por la sociedad que la cobija, de manera que actualmente se puede observar cómo al llegar una cultura dominante al entorno de otra con menos poder que además no tiene peso sobre el lenguaje global actual, como podría ser el inglés, la cultura dominante ejerce una influencia sentida sobre las prácticas, creencias, perspectivas, etc. (Atkinson, 1999; Holliday, 1999). De modo que se tienden a homogenizar las prácticas culturales y la naturaleza multicultural de las sociedades contemporáneas es ignorada por el mundo (Byram y Field, 2004).

Asimismo, Arabski (2011) conceptualiza la cultura como: "sum total of ways of living, including behavioral norms, linguistic expressions, styles of communication, patterns of thinking, and beliefs and values of a group large enough to be selfsustaining transmitted over the course of generation" (pp 26-27). Además, en el caso de las experiencias con culturas extranjeras, podemos ver que los académicos dicen lo siguiente:

A disheartening implication emerging from our discussion is that learners who study exclusively in their home country may feel that their language learning achievements lack validity, they may see themselves as the second class citizens of the language classroom and their abilities as somehow inferior to those whose language learning has been authenticated by an extended period overseas. 


\section{Relación cultura y lenguaje}

Además, Moran (2001) afirma que "el lenguaje es un producto de la cultura". De manera que los miembros de determinada cultura pueden compartir y llevar a cabo sus prácticas, identificar y organizar sus "productos" y así también poder darle lugar a las perspectivas que son originarias de su propia cultura. Así es que Moran (2001) asevera que: "... the words of the language, its expressions, structures, sounds, and script reflect the culture just as the cultural products and practices reflect the language. Language, therefore, is a window to the culture" (p. 37). Por lo cual la relación entre la cultura y el lenguaje queda establecida desde tiempos inmemorables, dándole cabida al fomento de un lenguaje global.

Así es que en este caso el inglés es visto como lengua extranjera, en tanto que no es hablada ni accesible en un sentido nativo a un gran número de personas dentro de los países tratados. Murray (2011) agrega un concepto interesante, considerando cómo es que la cultura en la que los miembros de una comunidad participan, puede establecer relaciones sociales que más que permitirles mediar sus acciones, también logra contribuir en el proceso de identidad y pertenencia dentro de dicha cultura. Además, define el contexto relevante para el análisis de la influencia de la cultura en ambos grupos de poblaciones en cuanto al aprendizaje del inglés, como el área o ambiente físico en el cual los individuos están localizados, así como el contexto socio-histórico en el que han sido educado o criados (Murray, 2001).

Es importante comprender como docentes y estudiantes de una lengua extranjera, como el inglés, que entender el lenguaje nos permite entender la cultura y viceversa. Así que cuando tenemos la posibilidad de asimilar el lenguaje o estar en contacto con la cultura, tenemos entrada directa para reconocer las creencias, valores y demás prácticas. De esa manera el lenguaje y la cultura no están separadas ni pueden estarlo, son adquiridas juntas, como lo manifiesta Arabski (2001) en su texto acerca de los aspectos de la cultura:

In order to communicate across culture, it is vital to comprehend cultural differences that may facilitate or debilitate communication. The cultural background of the interlocutors appears to have a serious influence on the quality and quantity of the communication act. Members of different societies when interacting need to understand cultural differences that are likely to obstruct their communication process. 


\section{Concepto de motivación}

Según Ana María Gálvez Fernández (2006), quien cita a varios autores dentro de su estudio acerca de la motivación, la define como un estado o condición interna que activa, dirige y mantiene un comportamiento (Schunk, 1996). Solana (1993), a su vez habla de la motivación en los siguientes términos:

La motivación es, en síntesis, lo que hace que un individuo actúe y se comporte de una determinada manera. Es una combinación de procesos intelectuales, fisiológicos y psicológicos que decide, en una situación dada, con qué vigor se actúa y en qué dirección se encauza la energía.

Generalmente el aprendizaje de una lengua extranjera es mejor cuando al estudiante le gusta no solo lengua a aprender sino también la cultura de la que este idioma hace parte de manera que desea familiarizarse e integrarse por medio del uso este.

Koontz y Weihrich (1999) la definen de la siguiente manera:

La motivación es un término genérico que se aplica a una amplia serie de impulsos, deseos, necesidades, anhelos, y fuerzas similares. Decir que los administradores motivan a sus subordinados, es decir que realizan cosas con las que esperan satisfacer esos impulsos y deseos e inducir a los subordinados a actuar de determinada manera.

Brophy, (1998), citado por Merlin (2003), define la motivación para aprender como:

Una red de razonamientos, valores, habilidades y disposiciones que permiten al alumno entender lo que significa comprometerse en actividades científicas con la intención de lograr sus metas de aprendizaje y al mismo tiempo estar consciente de las estrategias que usa para lograrlo. 


\section{Motivación integrativa}

Kruglanski (1975), citado por Bañuelos (1990), establece que "cuando se está comprometido con la tarea como tal se busca dominar el conocimiento, se aprende en las oportunidades que se presentan; el aprendizaje es libre, placentero y se convierte en un atributo endógeno". Es decir cuando un estudiante que tiene la capacidad se dedica al estudio del inglés posiblemente las habilidades que adquiera se convertirán en interés que lo impulsarán a ser autónomo en su aprendizaje.

La motivación integrativa relacionada con el aprendizaje de la lengua extranjera, típicamente implica la adquisición exitosa de una amplia gama de registros y de pronunciación muy parecida a la nativa (Finegan, 1999, citado por Norris, 2002). Benson (1991, pp. 34-38) sugiere que un enfoque más apropiado al concepto de motivación integrativa en el contexto del aprendizaje del idioma inglés como lengua extranjera sería la idea de que representa el deseo del individuo de hacerse bilingüe y bicultural a la vez. Es decir, el individuo adapta la cultura del idioma extranjero a su propia realidad y la integra a su forma de ser y de pensar. Podemos también mencionar aspectos personales que tienen que ver con el gusto y disfrute de aprender el idioma, tales como el placer de leer y disfrutar de formas de entretenimiento en inglés (Benson, 1991, pp. 36, citado por Norris, 2002). Además se tiene en cuenta la importancia del concepto de motivación integradora propuesta por Gardner (1985) en lo que al aprendizaje del inglés se refiere, ya que su importancia como lengua meta se incrementa cada vez más, en la medida en que el mundo globalizado se hace presente en todos los individuos que requieren de este idioma como medio de comunicación.

\section{Motivación instrumental}

Este tipo de motivación ocurre cuando el individuo que estudia el idioma inglés lo hace con la firme intención de conseguir una habilidad que producirá un beneficio práctico y es consecuencia de su imposibilidad de interactuar en un medio donde se hable el idioma que desea aprender. Se introduce el concepto de "requisito motivacional" con el que se refiere a aquellas actividades que los estudiantes tienen que realizar, no son opcionales para ellos ni de libre elección y que generalmente no son de su agrado, pero que terminan por motivarles a aprender la lengua extranjera de 
manera exitosa como lo serían los exámenes de estado como requisito de ingreso a la educación superior o de graduación.

Ushioda (2008), entiende motivación como "emergent through complex interactions among social, individual and contextual processes" (p. 25). Esta perspectiva de motivación demanda que se entienda claramente la relación entre motivación y las identidades sociales que emergen, las cuales toman lugar por medio de los procesos sociales pertenecientes a las múltiples comunidades de prácticas. De manera que la motivación no es individual, pero en su lugar es mediada por el interés y deseo social de pertenencia a los actos discursivos de un grupo social particular o institución. Además, de acuerdo con Wilkinson (1998), un periodo de tiempo extenso que se pase en un ambiente donde se hable la lengua extranjera, en este caso el inglés, tiene una alta incidencia en el aprendizaje y afecta positivamente, de acuerdo con (Freed, 1995, 1998) el nivel de motivación y de interés por el lenguaje.

\section{Motivación como factor que contribuye al aprendizaje de la lengua extranjera}

El trabajo llevado a cabo por Gardner en el área de la motivación fue en gran medida influenciado por Mowrer (1950, citado en Larson-Freeman y Larga 1994), cuyo foco fue la adquisición de la primera lengua. Mowrer (1950) propuso que el éxito de una persona en el aprendizaje de una primera lengua podría atribuirse al deseo de obtener la identidad dentro de la unidad familiar y la comunidad lingüística más amplia. Usando esto como base para su propia investigación Gardner pasó a investigar la motivación como un factor que influye en la adquisición de L2.

De este modo Gardner (1982), en su modelo socio-educativo, identificó una serie de factores que se interrelacionan en el aprendizaje de un segundo idioma. Estos factores son el entorno social y cultural, las diferencias individuales del alumno, el entorno o contexto en el que tiene lugar el aprendizaje y los resultados lingüísticos. El medio social o cultural se refiere al ambiente en el cual un individuo se encuentra, determinando así sus creencias acerca de otras culturas y de otros idiomas. Son estas creencias que tienen un impacto significativo en el aprendizaje de un segundo idioma. Gardner (1979, citado en Skehan, 1993) sugiere que las expectativas relativas al bilingüismo, en combinación con las actitudes hacia la lengua extranjera y su cultura, forman la base de la actitud de un individuo hacia el aprendizaje de idiomas. 
La segunda fase del modelo de Gardner presenta las cuatro diferencias individuales que se cree son los más influyentes en el aprendizaje de un segundo idioma. Estos incluyen las variables de la inteligencia, la aptitud lingüística, la motivación y la ansiedad situacional (Giles y Coupland, 1991). Estrechamente relacionados entre sí con estas variables se presenta la siguiente fase del modelo, denominado el entorno o contexto en el que tiene lugar el aprendizaje. Dos contextos se identifican, es decir, la instrucción formal en el aula y la adquisición del lenguaje estructurado en un entorno natural.

La fase final del modelo identifica los resultados lingüísticos que se refieren a los conocimientos y habilidades lingüísticas, idioma reales, y los resultados no lingüísticos de la experiencia de aprendizaje que reflejan las actitudes de un individuo en relación con los valores culturales y sus creencias, por lo general hacia la comunidad idioma de destino (Ellis, 1997). Esto ocurre a través de la aprehensión de otra lengua y de la cultura a la identidad cultural propia del alumno.

\section{Motivación integrativa versus instrumental en el aprendizaje de la lengua extranjera}

En contraste con la motivación integradora, la motivación instrumental se caracteriza por el deseo de obtener algo práctico o concreto a partir del estudio de un segundo idioma (Hudson 2000). En cuanto a la motivación instrumental el propósito de la adquisición del lenguaje es más utilitario, como el cumplimiento de los requisitos educativos o la graduación de la universidad, solicitar un trabajo, solicitar mejor remuneración basada en la capacidad del lenguaje, la lectura de material técnico, el trabajo de traducción o la consecución de un mayor estatus social. La motivación instrumental es a menudo característica del aprendizaje de segundas lenguas, donde poco o nada de la integración social del alumno en una comunidad que utiliza el idioma de destino se lleva a cabo.

En algunas de las primeras investigaciones realizadas por Gardner y Lambert, la motivación integradora fue vista como un aspecto de mayor relevancia en un ambiente de aprendizaje formal más que la motivación instrumental (Gardner y Lambert, 1972, p. 12). Se ha encontrado que los estudiantes seleccionan generalmente razones instrumentales con mayor frecuencia que las razones de integración para el estudio del lenguaje. Los que apoyan un enfoque integral para el estudio del idioma suelen estar más altamente motivados y en general tener mayor éxito en el 
aprendizaje de idiomas. Brown (2000) apunta que tanto la motivación integradora como la instrumental no son necesariamente excluyentes entre sí. Los estudiantes raramente tienen una sola forma de motivación en el aprendizaje de un segundo idioma, sino más bien una combinación de ambas orientaciones.

La motivación es un factor importante en el aprendizaje de la L2. Por esta razón, es importante identificar el tipo y combinación de motivaciones que ayuda a la exitosa adquisición de esta. Al mismo tiempo es necesario ver la motivación como una de las variables en un modelo complejo de factores individuales y de situaciones interrelacionadas que son únicos para cada estudiante de idiomas. El aprendizaje de la lengua extranjera y la motivación han sido recientemente relacionados con las teorías socioculturales donde el aprendizaje es visto como un fenómeno social mediado.

\section{Metodología}

Esta investigación es de carácter mixto porque al reunir las características del enfoque de investigación cualitativo y cuantitativo permitió además de analizar en un mismo momento desde diferentes perspectivas, los modos en los que se ve influenciada la motivación de los universitarios de las dos instituciones elegidas, en relación con la cultura y algunos aspectos sociales se logró recolectar datos con el fin de realizar una medición numérica que acompañó el análisis de manera estadística para establecer aquellos patrones en las respuestas obtenidas.

Grinnell (1997), citado por Hernández et al. (2003, p. 5) señala que los dos enfoques (cuantitativo y cualitativo) utilizan cinco fases similares y relacionadas entre sí, lo que permite que ambos puedan ser usados en la misma investigación:

- Llevan a cabo observación y evaluación de fenómenos.

- Establecen suposiciones o ideas como consecuencia de la observación y evaluación realizadas.

- Prueban y demuestran el grado en que las suposiciones o ideas tienen fundamento.

- Revisan tales suposiciones o ideas sobre la base de las pruebas o del análisis. 
- Proponen nuevas observaciones y evaluaciones para esclarecer, modificar, cimentar o fundamentar las suposiciones o ideas; o incluso para generar otras.

Es además, un estudio comparativo porque los datos recolectados se cotejan para hallar las similitudes, diferencias y posibles acciones acordes con el proceder de una comunidad con respecto a la otra, con el fin de mejorar los procesos de aprendizaje de una lengua extranjera, por un lado, y por el otro, informar y promover que los estudiantes puedan hacer uso de estas motivaciones y de la posible influencia de la cultura en su futura profesión docente.

\section{Participantes}

Se encuestaron en total 49 estudiantes de educación superior, entre ellos 23 estudiantes de la Universidad Mayor de Chile y 26 estudiantes de la Universidad Distrital Francisco José de Caldas de Colombia, todos pertenecientes a séptimo semestre, cuarto año de Pedagogía Licenciatura en Inglés. Entre el total de estudiantes encuestados (ambas universidades) el número de mujeres participantes fue de 31, 15 hombres y 3 personas que prefirieron no dar esta información. Asimismo, la media dentro del nivel de inglés que los participantes consideran tener estuvo entre intermedio y avanzado, teniendo en consideración que 13 de ellos no especificaron esta información. Los estudiantes de la UD son jóvenes de 19 a 22 años de edad y los estudiantes de la UM están en edades entre 21 y 25 años, con predominancia alumnos de 22 años de edad, dos años más en comparación con el mayor porcentaje de la UD (véase las figuras en los anexos).

\section{Resultados}

El primer resultado que se obtiene y que da respuesta a la pregunta de investigación, es que el entorno socio cultural tiene gran incidencia en el desarrollo y mantenimiento de la motivación para el aprendizaje de una lengua extranjera en alumnos de séptimo semestre de Pedagogía en Inglés de la Universidad Mayor de Chile, y en los estudiantes del mismo semestre de Licenciatura en Inglés de la Universidad Distrital, ya que por medio de los planes de bilingüismo que se están implementando últimamente en ambos países y de acuerdo con los resultados obtenidos por 
medio de los instrumentos de investigación, los estudiantes tienen actualmente la posibilidad de tener contacto con la cultura de la cual se está aprendiendo, lo que genera una especial motivación en los estudiantes por el conocimiento de las culturas anglosajonas, además aspectos que son importantes para motivar a los estudiantes, de acuerdo con lo analizado, es la música, la literatura y el cine, que llaman demasiado la atención de los estudiantes dentro y fuera de los entornos académicos y además hacen parte importante de la vida de cada uno de ellos y de la cultura propia.

Otro punto interesante es el notorio interés de los estudiantes por hacer parte de esa cultura global en la que el inglés es el principal medio para tener una participación activa, que más allá de estar relacionado con la globalización les da la posibilidad de tomar parte dentro de diferentes grupos culturales, académicos, de intereses comunes conformado por personas de cualquier parte del mundo. Esto refuerza el uso del inglés como lengua franca (Universia, 2013), lo que permite la solución de problemáticas y también como medio de interacción y de intercambio cultural dentro de los diferentes contextos y espacios que se le abren (centros educativos, actos culturales y artísticos, política, economía, etc.), lo que directamente motiva a los estudiantes a iniciar su carrera como licenciados o pedagogos, según sea el caso, de inglés como lengua extranjera.

Los resultados obtenidos con la encuesta realizada permitieron dar a conocer diferentes aspectos que responden y aportan al desarrollo de la presente investigación, aunque quizá no fueron los esperados, fueron lo más honestos y cercanos a la realidad que se pudo haber obtenido (véase las figuras anexas al final del documento).

De acuerdo con la definición de cultura aportada por Patrick Morán (2001) (véase en el anexo: Encuesta [1 pregunta]) y con la cual el $96 \%$ de los estudiantes de la UM y el $92 \%$ de los encuestados de la UD están de acuerdo. Además de obtener estos resultados, los encuestados aportaron su propia visión o percepción acerca del concepto de cultura, así es que de acuerdo con sus aportes, los estudiantes encuestados de la Universidad Distrital consideraron que además de que es un conjunto de prácticas asociado con los productos y los puntos de vista compartidos entre los miembros del grupo quienes están dentro de contextos específicos en los que han sido formados (Murray, 2001); la cultura se transforma, cambia a medida que transcurre el tiempo, está definida por un tiempo y épocas determinadas, así como lo plantea Cavalli (2007), la cultura es una agrupación de conocimientos que como individuos traspasamos de generación en generación, 
pero que al ser compartida tiende a ser transformada. Además, consideran que aportan una identidad que es compartida y muchas veces adquirida, aprendida y asumida (Levy, 2014) por Colombia como país no angloparlante por causa de la interacción actual con la cultura de países con esta lengua.

En el caso exacto de los estudiantes de Pedagogía en Inglés de la UM de Chile, ellos consideran que además debería sumársele a esta definición que no hay una sola cultura, sino múltiples culturas que dejan un precedente a través de la historia. Que así como lo planteaba anteriormente Cavalli (2007) esta se entrega, en palabras usadas por los encuestados, como herencia de una generación a otra. Además, se cree necesario analizar el hecho de que aunque fue solo uno de los participantes en cada una de las universidades (dos en total) quienes pidieron replantear la definición de cultura dada porque consideran que el término evolutiva usado en esta hace parecer la cultura como algo que está o requiere continuo mejoramiento.

Acorde a las respuestas de los estudiantes de la UM, el aspecto de la cultura que más le motiva a estudiar inglés es el posible intercambio cultural que se puede obtener como beneficio de manejar la lengua. Además, se hace énfasis en la interacción global como factor motivante, esto es asociable con el uso reciente del inglés como lengua franca (Universia, 2013), lo cual permite que las personas que lo manejan tengan la oportunidad de participar dentro de las comunidades globales interculturales, en donde los miembros de las mismas no tienen el inglés como lengua materna sino que esta se usa como medio para compartir lo que se desee, creencias, perspectivas de vida, estilos, etc. Además, los estudiantes muestran gran interés en lo que son las artes, la literatura, el cine y la música, especialmente esta última, pues son parte de los aspectos culturales que motivan a los estudiantes porque tienen afinidad con sus gustos personales; de modo que los estudiantes ven que la cultura, en general, sin especificar una de ellas, motivan el aprendizaje del inglés a nivel global, por los beneficios que este le puede traer a una persona a nivel laboral, estatus social, poder y propósitos del lenguaje de acuerdo con la motivación instrumental que se abordó anteriormente.

Para esta segunda inquietud, el principal factor de motivación proveniente de una cultura según los estudiantes de la Universidad Distrital, también es la interacción cultural que puedan tener como resultado de sus estudios de la lengua extranjera; así también, se mencionan aspectos como los que reciben de las culturas angloparlantes, que por ser diferentes a la propia y diversas entre ellas captan la atención de los estudiantes y les fomenta el deseo por el aprendizaje de la lengua. En común con los resultados provenientes de los alumnos de la UM, además se 
reconoce el rol de las artes, pero en este caso con más énfasis en el cine, en el cual varios de los encuestados consideran tener bases de conversación y puntos de discusión que permiten y proveen esos encuentros interculturales. El peso de aspectos como la globalización, el inglés como requerimiento laboral es más fuerte en el caso colombiano teniendo en cuenta las respuestas de los participantes, aunque estos son más reacios a la asimilación y participación dentro del sistema; sin embargo reconocen la actual importancia de este hecho y los beneficios que recaen sobre ellos al saber inglés, lo que hace alusión a la motivación instrumental que les insta a cumplir con el requerimiento social actual.

La pregunta número tres se enfocó en el nivel de facilidad para aprender la lengua extranjera por parte de los estudiantes, por lo cual los resultados de acuerdo con lo esperado no fueron negativos, es decir, no hubo ningún participante proveniente de ninguna de las dos universidades analizadas que manifestara tener poca facilidad para estudiar el idioma. Este aspecto de la encuesta permite reflexionar acerca de la motivación personal, intrínseca de los estudiantes, la voluntad de cada uno de ellos por aprender la lengua de acuerdo con los fines que cada uno de ellos le dé a la misma (Ramo, 2003). De esta manera, el estudiante tiene la posibilidad de trabajar el idioma desde su propia perspectiva del mismo, desde su nivel de facilidad o dificultad para aprenderlo.

Aunque la intención de la pregunta número cuatro fue conocer cuáles eran los tres principales motivos para el aprendizaje de inglés de los encuestados, aquí se dará esa apreciación a nivel global, ya que las similitudes y variaciones entre las respuestas no fueron muy significativas. Así es que el motivo más importante para los participantes (teniendo en cuenta que cada participante dio tres posibles motivos) es el poder enseñar la lengua luego de su correspondiente aprendizaje e internalización. El segundo es, según el número de participantes que optaron por este, el interés personal por viajar y conocer la cultura de habla inglesa, lo que hace referencia a la interacción social de la que anteriormente se hablaba como motivante para los estudiantes de pregrado en Pedagogía y Licenciatura de inglés. Por último, y no menos importante, en el ranking de motivaciones encontramos el gusto personal por este idioma en específico, lo que suma puntos a la motivación intrínseca de los estudiantes por culminar sus procesos de aprendizaje. Algunos otros motivos por los cuales los estudiantes aprenden la lengua son, nuevamente su interés personal y la búsqueda de nuevas perspectivas y puntos de vista ante la vida.

Solo el $14 \%$ del total de los participantes de ambas universidades, es decir, 7 estudiantes de 49 encuestados han tenido la oportunidad de realizar una inmersión 
en un país angloparlante. Entiéndase inmersión conforme al concepto establecido por Alarcón Neve (2002):

Inmersión es un contacto intervenido por la escolarización. Por ejemplo, lo que la minoría de participantes han realizado trasladándose a otro país en donde tienen contacto con nativo hablantes y a la vez una guía o formación académica en su proceso de adquisición de la lengua extranjera (p. 127).

Además, los estudiantes que respondieron afirmativamente dieron su opinión acerca de la manera en la que influyó esta inmersión en su proceso; manifestaron haber, como se espera, mejorado sus niveles de adquisición y competencia del inglés, reconocer nuevos puntos de vista y perspectivas culturales que ampliaron su modo de vida y de concepción del mundo circundante. Sin embargo, estos estudiantes también mencionaron el tiempo como factor influyente en los beneficios que pudiesen obtener de su paso por países angloparlantes. Es de tener en cuenta que de acuerdo con Wikinson (1998) y los datos recolectados por medio del instrumento de investigación, el periodo de tiempo que se viva inmerso en un país angloparlante, permitirá e incidirá en el aprendizaje del inglés del estudiante, y así mismo afecta positivamente aumentando su motivación e interés por la lengua extranjera, en este caso el inglés. Es también importante tomar en consideración el número tan alto de estudiantes que por diferentes y desconocidas razones para nosotros no han tenido la oportunidad de acceder a un intercambio de este tipo, nos hace preguntarnos cuál es el rol de los programas de movilidad académica en la formación de licenciados y pedagogos con énfasis en inglés. En las dos universidades, que no propician unos niveles más altos de competencia lingüística y comunicativa, con el propósito de mejorar los niveles de lengua de los niños y niñas que los mismos al egresar de las universidades tendrán como sus estudiantes.

Para continuar se les preguntó a los estudiantes si su cultura, la de Chile o Colombia según fuera el caso, motivaba el aprendizaje del inglés. En Colombia, los estudiantes en su gran mayoría consideran que sí, aunque lo relacionan directamente con la influencia que ejercen las culturas angloparlantes sobre la de nuestro país, a lo que podemos hacer alusión desde lo que Atkinson (1999) considera como que una cultura dominante ejerce una influencia sentida sobre las prácticas, creencias, perspectivas, entre otros, de manera que se intentan igualar las prácticas de la cultura, en este caso colombianas, a las de los países angloparlantes 
siguiendo los discursos de poder con los que estos se presentan. Además, se tiene muy en cuenta el papel que hoy juega el inglés como medio para tener acceso a mejores oportunidades de trabajo, lo que algunos de los estudiantes colombianos ven como motivación y otros como "requisito motivante" que en realidad no le da al sujeto la posibilidad de elección, sino que en su lugar le impone el tener que demostrar y recientemente, certificar su nivel de dominio del idioma extranjero. En el caso chileno, los estudiantes reconocen que hay cierta motivación generada por su cultura en tanto que se adoptan costumbres y modos de vida que les hacen cercanos a culturas angloparlantes; asimismo, consideran que hace falta fomentar el conocimiento de los beneficios sociales, culturales, económicos, etc. que el inglés puede generarles. La globalización adoptada por su cultura, también es considerada como un factor motivante para el aprendizaje de la lengua.

Con el propósito de reconocer la influencia de las culturas angloparlantes, que según los estudiantes, es ejercida en sus propias culturas, se les encuestó acerca de sus percepciones acerca de los países angloparlantes; es importante tener en cuenta que la mayoría de ellos lo hicieron teniendo como referente países como Inglaterra, Estados Unidos, Australia; así, los estudiantes colombianos en su mayoría consideran positiva e influyente la percepción que tienen de estos países. Ellos afirman que además de ser una cultura más organizada, mejor, más interesante y que les brinda unas perspectivas de vida de mayor calidad, es una cultura desarrollada que aporta conocimiento, desarrollo tecnológico y científico, entre otros; lo anterior es influyente en su decisión de aprender inglés para luego enseñarlo. En cuanto a los estudiantes chilenos, ellos también les consideran más desarrollados, con diferentes modos de vida y de interacción que los hacen tradicionales y que han dejado más huella en la historia del mundo. Asimismo, los estudiantes chilenos en su mayoría tienen una percepción positiva de estos países y les consideran influyentes en su proceso de aprendizaje. Solo algunos de los estudiantes encuestados, una minoría, considera negativa y no influyente su visión de la cultura en estos países además de que le atribuyen o las califican como culturas consumistas, dominantes e individualistas. Con esta pregunta es posible ver la oportunidad que tenemos los docentes para motivar a los estudiantes a estudiar inglés como lengua extranjera, como lo aseveran y comparten Cheung, 2001; Duzz, 2002, 2004; Marsh, 2005; Moffatt y Norton, 2005; citados por Murray (2001), quienes como académicos han promovido el uso de una cultura popular con el propósito de motivar e incentivar a los estudiantes en el aprendizaje de la lengua. 
Una duda que surgió al realizar la presente investigación fue si los estudiantes de pedagogía de la UM y los estudiantes de Licenciatura en Inglés de la UD quieren convertirse en docentes de inglés al terminar su formación. Así es que el $100 \%$ de los estudiantes de la UM desean ser docentes al finalizar la carrera, en cambio en la UD solo el $92 \%$ de los estudiantes quieren ejercer la docencia al salir de su pregrado. Algunas de las razones que los estudiantes de ambas instituciones manifiestan para tomar la decisión de ser profesores son: mostrarle a los estudiantes la actual importancia del inglés dentro de ambas culturas, además de que consideran que por medio de la educación se puede lograr una verdadera transformación social; otro aspecto importante que se menciona en las respuestas de los participantes es que es parte de su vocación el impartir conocimientos y de esa manera enriquecer su proceso personal y asimismo ser de ayuda para quienes lo necesitan. Algunos de los estudiantes, el $4 \%$ del total de los participantes, manifestó no querer ser docentes al salir de sus estudios de pregrado porque lo que quieren es realizar estudios de posgrado o de educación avanzada en donde el manejo del inglés les es requerido.

Para concluir, acorde a los resultados alcanzados con esta investigación es que los estudiantes de Pedagogía en Inglés de la UM de Chile y los estudiantes de Licenciatura en Inglés de la UD de Colombia, se sienten motivados a estudiar inglés como lengua extranjera con el objetivo primordial, en su mayoría, de generar procesos de mejoramiento y transformación social, así como también su ferviente interés como estudiantes y futuros docentes por cambiar los imaginarios de las personas alrededor del mundo acerca de su cultura nativa, lo que nos permite ver que les motiva más a aprender una lengua extranjera por lo que pueden hacer con ella en su cultura. Además de considerar muy bajo el nivel de motivación que ejerce su cultura propia, las percepciones de la población investigada tienden a minimizar el impacto de esta sobre los procesos que llevan a cabo para darle paso a sus intereses por culturas anglosajonas.

Así, lo que termina por motivar a los estudiantes en el aprendizaje de una lengua extranjera es la motivación integrativa por encima de cualquier tipo de motivación de parte de su cultura, y acorde a lo establecido por los autores su propósito es más utilitarista, los estudiantes de ambas universidades manifiestan su interés por obtener beneficios personales por medio del aprendizaje del inglés como lengua extrajera dentro de sus contextos sociales y culturales, pero como ya se mencionó, 
sin que esto sea producto de la influencia de sus respectivas culturas, colombiana y chilena según sea el caso, en esta decisión.

\section{Referencias Bibliográficas}

Alarcón, L. J. (2002). Bilingüismo y adquisición de segundas lenguas: inmersión, sumersión y enseñanza de lenguas extranjeras. Simposio CONCYTEQ. Colección Pedagógica Universitaria. Universidad Autónoma de Querétaro.

Arabski, J. (2011). Aspects of culture in second language acquisition and foreign language learning. Berlin: Springer.

Atkinson, D. (1999). TESOL and Culture. TESOL Q 33(4), 625-654.

Bañuelos, A. (1993). Motivación Escolar: estudio de variables afectivas. En Revista Perfiles Educativos, 60. México.

Bañuelos, A. (1990). Motivación Escolar: una propuesta didáctica. En Revista Perfiles Educativos, 49. México.

Benson, M. J. (1991). Attitudes and motivation towards english: A survey of japanese freshmen. RELC Journal, 22(1), 34-48.

Brown, H. D. (2000). Principles of language learning and teaching (4th edition). Englewood Cliffs, N.J: Prentice-Hall.

CavalliSforza, L. L. (2007). La cultura y su evolución. La evolución de la cultura. Barcelona: Editorial Anagrama (trabajo original publicado en el 2004).

Chile, E. F. (2013, enero). Índice del nivel de inglés (tercera edición). Disponible en http:// www.ef-chile.cl/epi/

Crooks, G., \& Schmidt, R. W. (1991). Motivation: Reopening the research agenda. Language Learning, 41, 469-512.

EF EPI. (2013). Índice del nivel de inglés. EF. EF EPI EF English Proficiency Index, 3. Disponible en http://www.ef-chile.cl/_/ /media/efcom/epi/2014/full-reports/ef-epi-2013 -report-mx.pdf

EF EPI. (2013, enero). Tendencias del índice del nivel de inglés. España. Disponible en http://www.ef.com.es/epi/

Ellis, R. (1997). The study of second language acquisition. Oxford University Press.

Gálvez, A. M. (2006). Motivación hacia el estudio y la cultura escolar: estado de la cuestión. Pensamiento psicológico, (2), 87-101.

Giles, H., \& Coupland, N. (1991). Language: Contexts and consequences. Open University Press. 
Giroux, H. A. (1988). Teachers as intellectuals: Towards a critical pedagogy of learning. Granby, M.A: Bergin \& Gavey.

Hernández, R., y et al. (2003). Metodología de la investigación (tercera edición). México: McGraw-Hill.

Hudson, G. (2000). Essential introductory linguistics. Blackwell Publishers.

Kramsch, C. (1998). Language and culture. Oxford: Oxford University Press.

Koontz, H., y Weihrich, H. (1999). Administración, una perspectiva global (onceava edición). México: McGraw-Hill.

Kövecses, Z. (2006). Language, mind and culture: a practical introduction. Oxford: Oxford University Press, $400 \mathrm{~S}$.

Larson-Freeman, D., \& Long, M. H. (1994). An introduction to second language acquisition research. Longman.

Levy, M. (2007). Language Learning and Technology (2th edition). In Culture, Culture Learning and New Technologies: Towards a Pedagogical Framework, 11, 104-127. Griffith University.

Lindsay, R. B., Robins, K. N., \& Terrell, R. D. (1999). Cultural literacy: A manual for school leaders. Thousand Oaks: Corwin.

Moran, R. (2001). Teaching Culture: Perspectives in practice. Canada: Helen \& Helen.

Moorjani, A., \& Field, T. T. (1988). Semiotic and sociolinguistic paths to understanding culture. In A. J. Singerman (Ed.). Toward a new integration of language and culture. Reports of the Notrheast Conference on the Teaching of Foreign Languages, pp. 25-45. Middlebury conference.

Murray, G. (2011). Identity, motivation and autonomy in language learning. Bristol: Multilingual Matters.

Norris-Holt, J. (2001). Motivation as a Contributing Factor in Second Language Acquisition. TESL Journal, 7(6). Retrieved in http://iteslj.org/Articles/Norris-Motivation.html

Schunk, D. H. (1996). Motivation in Education: Current emphases and future trends. Mid Western Educational Researcher, 9, 5-11.

Solana, R. (1993). Administración de organizaciones. Buenos Aires: Ediciones Interoceánicas. Disponible en http://www.monografias.com/trabajos5/moti/moti.shtml\#desa

Taylor, D. M., Meynard, R., \& Rheault, E. (1977). Threat to ethnic identity and secondlanguage learning. In H. Giles, Language, ethnicity and intergroup relations. Academic Press.

Universia. (2013, enero). Estudios internacionales, sección especial. Disponible en http:// internacional. universia. net/seccionEspecial.jsp?idEspecial=315\&idSeccion=8036\&tit $l e=$ INGLES-LENGUA-FRANCA-SIGLO-XXI 
Ushioda, E. (2008). Motivation and good language learners. In C. Griffiths (ed.). Lessons from good language learners, 19-34.

Wilkinson, S. (1998). Study abroad from the participant's perspective: A challenge to common beliefs. Foreign Language Annals 31(1), 23-39.

\section{Anexos}

a. Nivel de dominio del inglés (estudiantes de ambas universidades).

\section{Nivel de Inglés (UD-UM)}

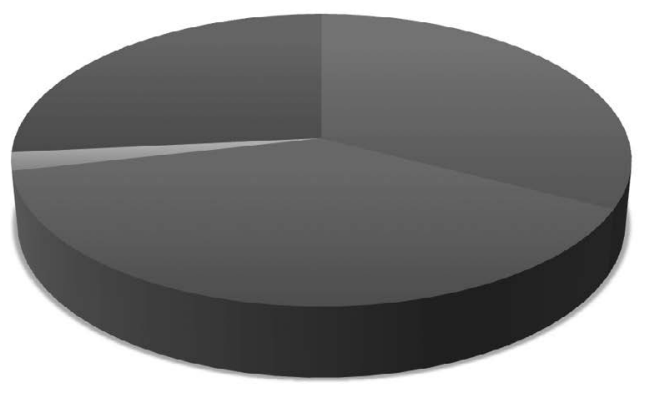

\section{- Avanzado}

- Intermedio-Superior

Básico

no Especifica

b. Nivel de proficiencia en inglés (estudiantes UD).

\section{Nivel de Inglés (UD)}

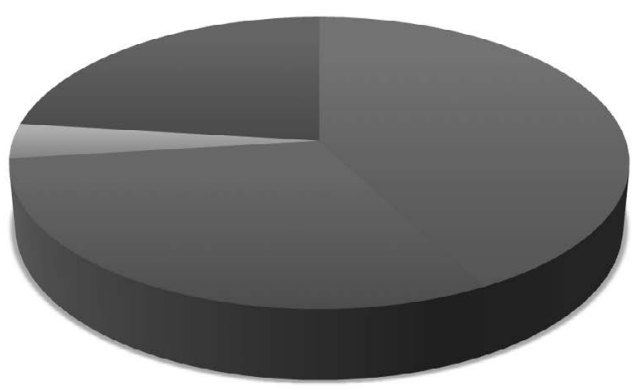

- Avanzado

- Intermedio-Superior

घásico

- No Especifíca 


\section{Nivel de Inglés (UM)}

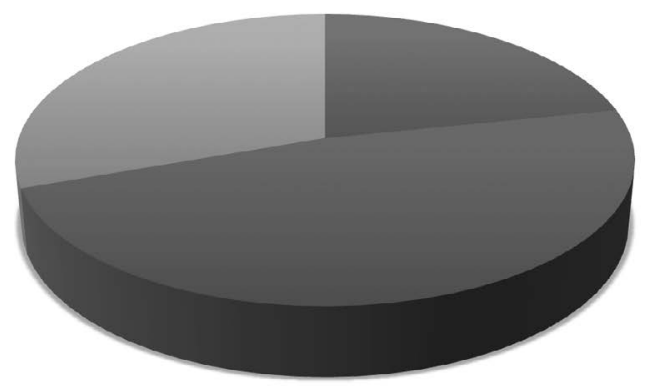

- Avanzado

- Intermedio-Superior

No Especifíca

d. Distribución de la muestra por género.

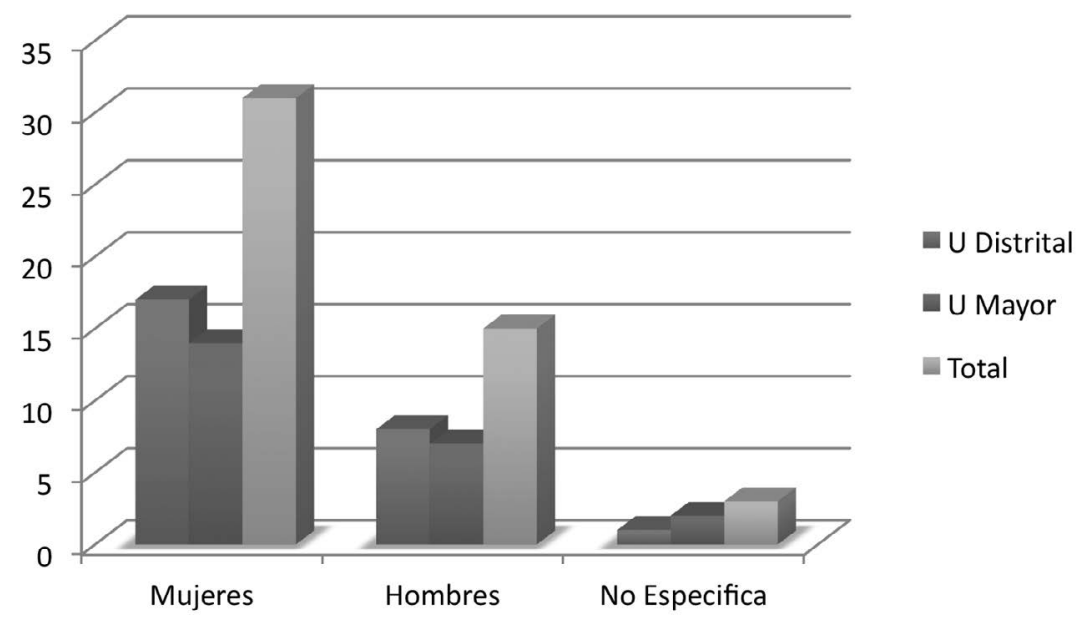


e. Distribución de la muestra por edades.

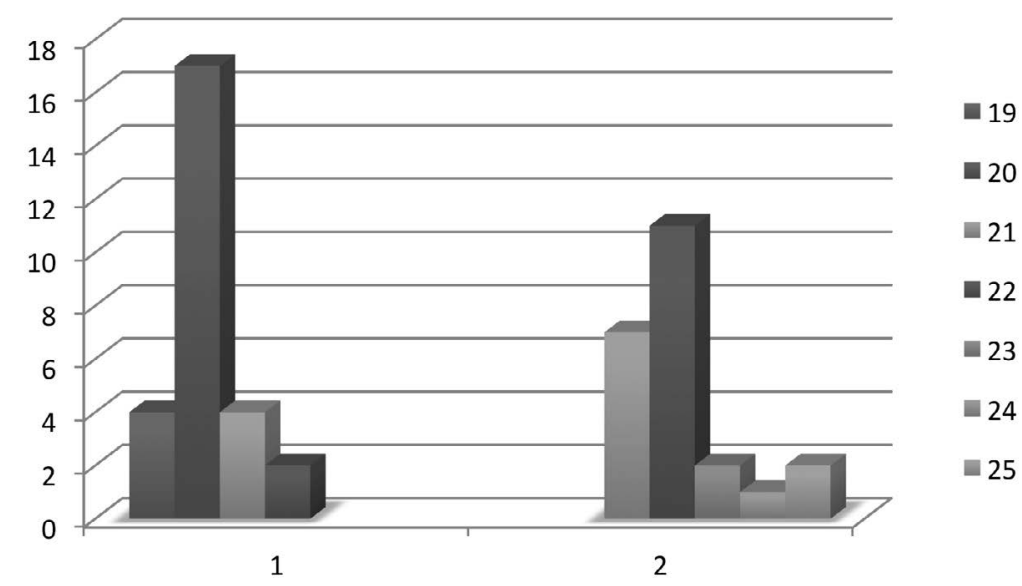

f. Colegios en los que estudiaron educación básica (UD-UM).

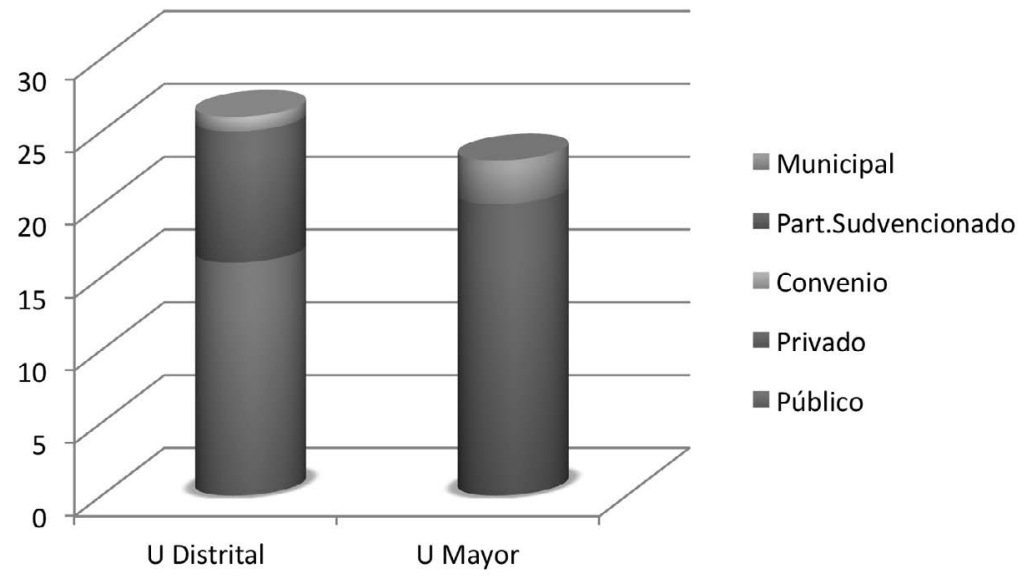


g. Colegios en los que estudiaron educación media (UD-UM).

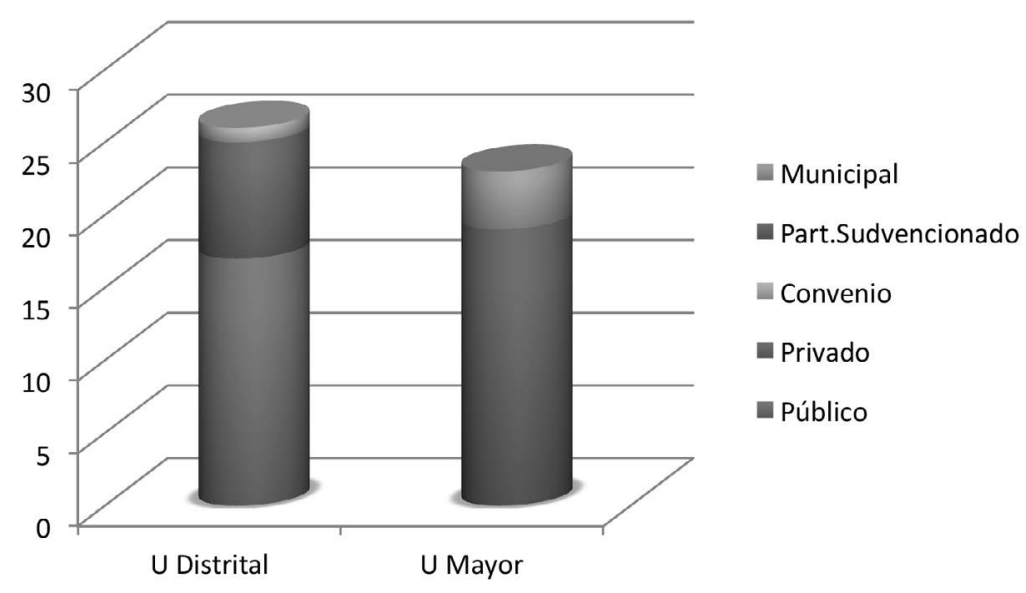

h. Encuesta aplicada.

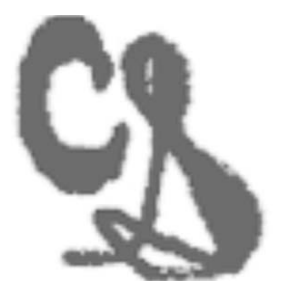




\section{Semillero de investigación calidad y saberes}

\section{Encuesta sobre motivación}

Semestre/año:

Nivel idioma:

Edad:

Universidad: Género:

Tipo de colegio en el que realizó la educación básica:

Tipo de colegio en el que realizó la educación media:

1. ¿Está usted de acuerdo con la siguiente definición de cultura? Si no es así, ¿qué omitiría o agregaría a la misma?

La cultura es el camino evolutivo de la vida de un grupo de personas, que consiste en un conjunto de prácticas asociadas a un conjunto de productos, con base a un conjunto de puntos de vista sobre el mundo compartidos entre los miembros del grupo, y que figuran dentro de contextos sociales específicos (Moran, Patrick. 2001).

2. ¿Qué aspectos de la cultura considera que motivan su aprendizaje del inglés como lengua extranjera? ¿Por qué?

3. ¿Cuál es su facilidad para estudiar inglés como lengua extranjera?

a. Buena

b. Regular

c. Mala

4. ¿Cuáles son los motivos por los que estudia inglés? Escoja las 3 opciones que considere son las más importantes. Tenga en cuenta que 1 representa la que para usted está en primer lugar. 
Para enseñarlo.

Me gusta el idioma inglés.

Me interesa viajar y conocer culturas de habla inglesa.

Para obtener mejores posibilidades de empleo.

Es un interés personal.

Para ampliar mi punto de vista y perspectiva.

Para entender películas, canciones, textos, etc.

Para realizar estudios de mayor formación (posgrado, maestría, especialización, etc.)

Para incrementar mi vida social.

5. ¿Ha viajado alguna vez a algún país en donde se hable inglés como lengua materna? De ser así, ¿de qué manera influyó esta vivencia en tu motivación para aprender inglés?

6. ¿Cómo considera que su cultura motiva su aprendizaje de una lengua extranjera como el inglés?

7. ¿Cuál es su percepción acerca de la cultura de los países angloparlantes? ¿Cómo influye esta percepción en su deseo por aprender inglés como lengua extranjera?

8. ¿Desea usted ser profesor de inglés al finalizar la carrera? ¿Por qué? 
9. ¿Cuáles de sus motivaciones para aprender inglés considera que son provenientes de la cultura a la cual pertenece?

i. Figura acuerdo o desacuerdo con el concepto de cultura (Moran, 2001).

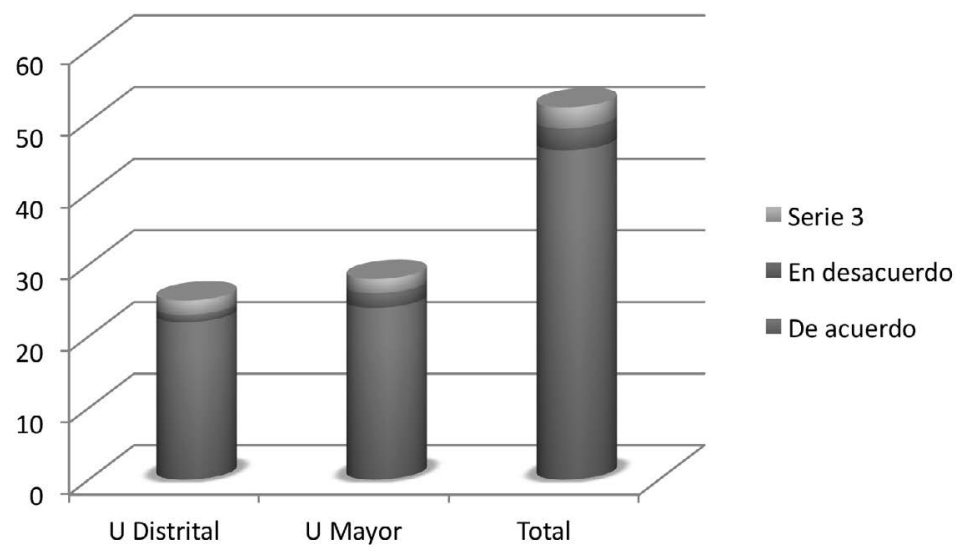

j. Figura aspectos culturales que motivan el aprendizaje del inglés.

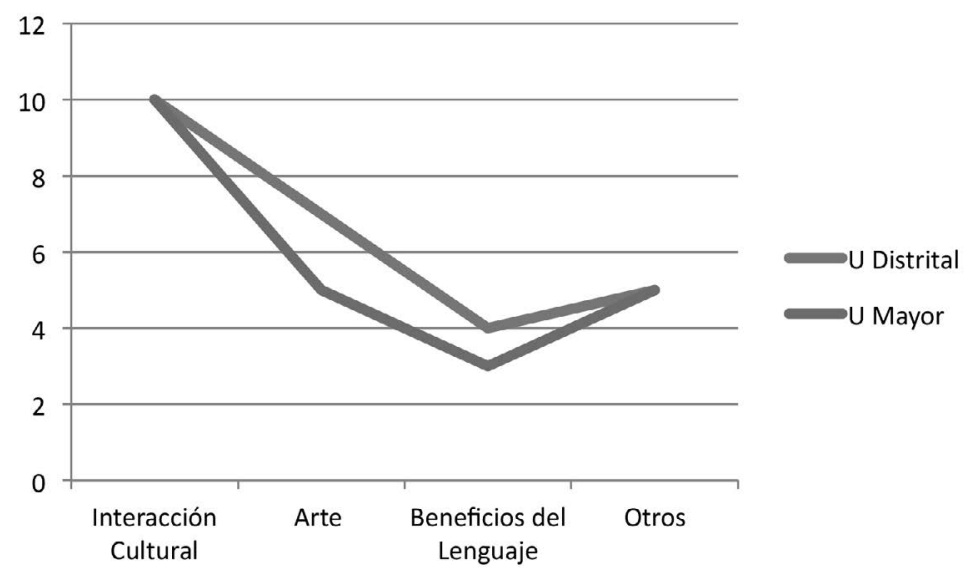


k. Figura facilidad de estudio del inglés.

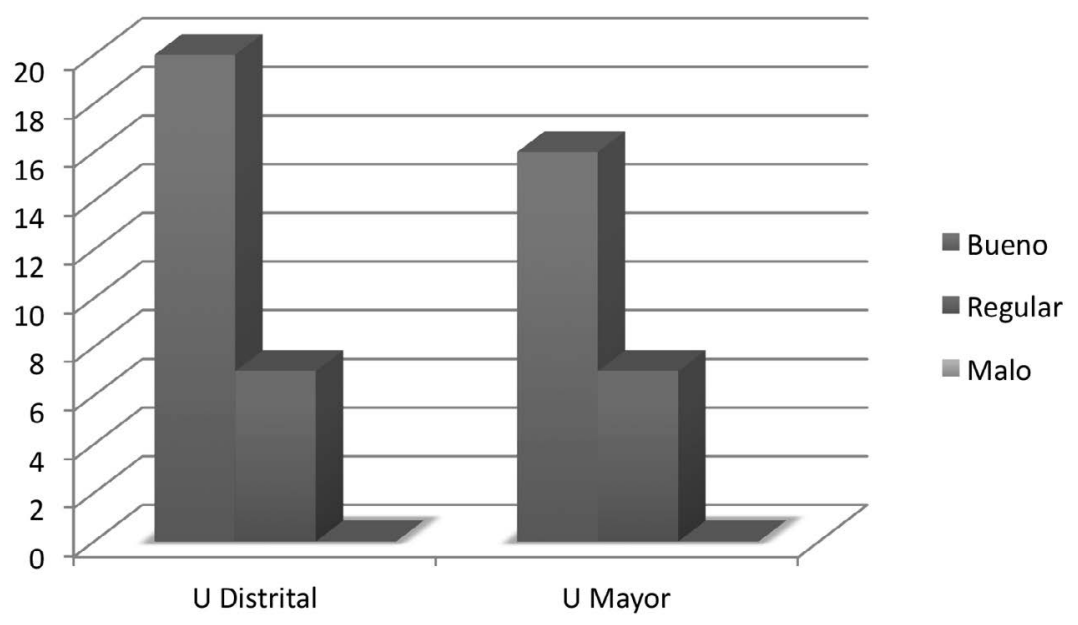

I. Figura motivos de aprendizaje del inglés.

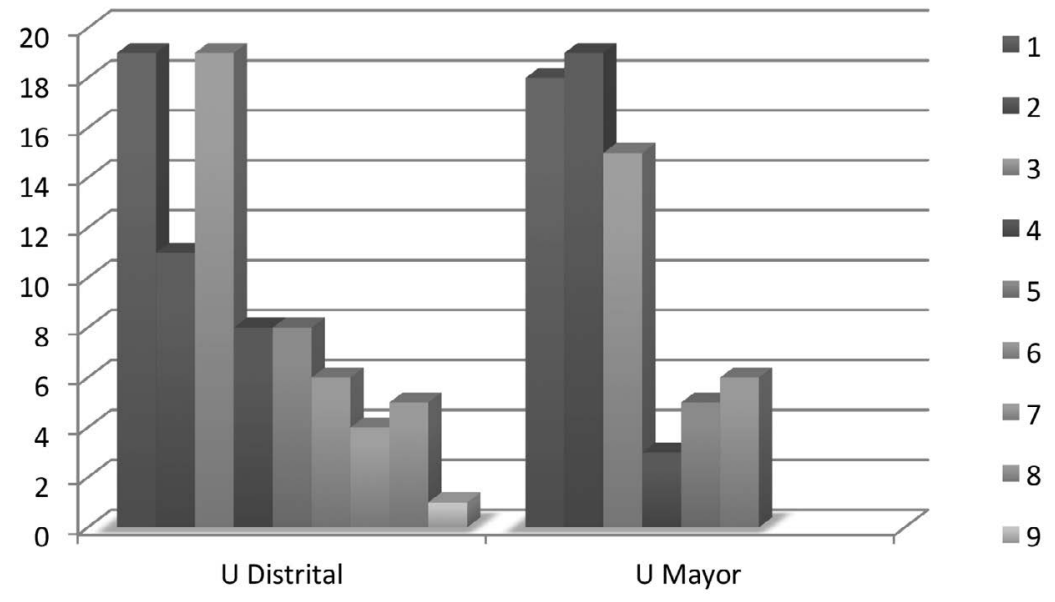


m. Figura ha viajado a un país angloparlante.

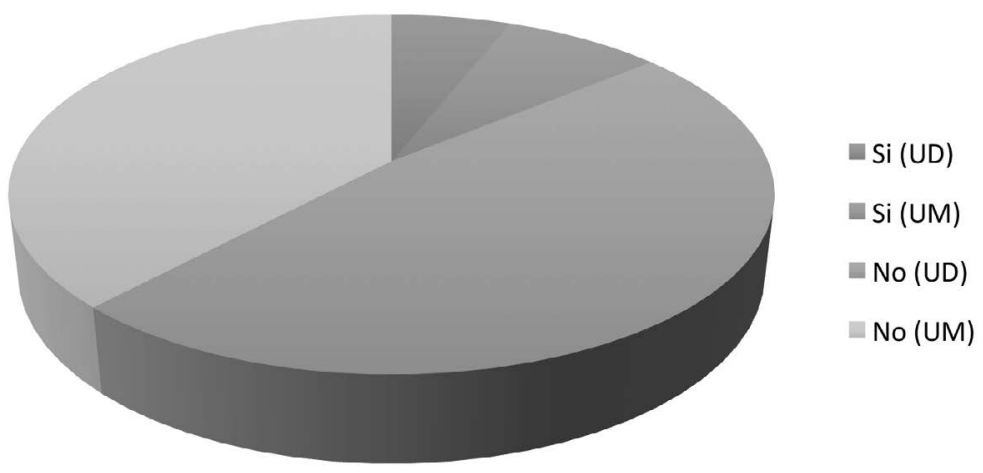

n. Figura motiva su cultura el aprendizaje del inglés.

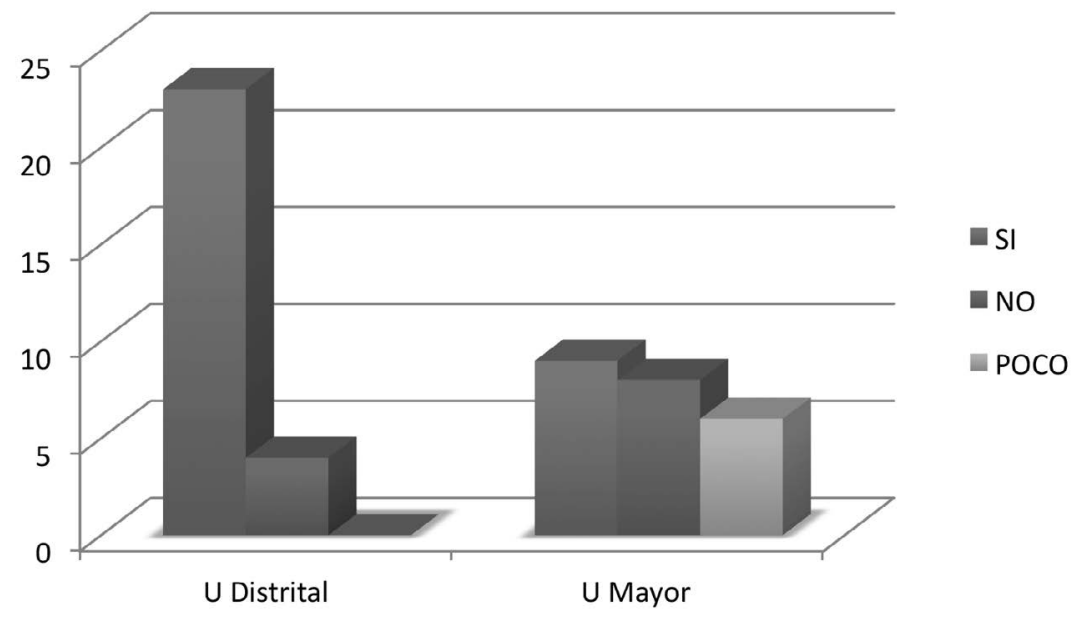


o. Figura percepción de los países angloparlantes.

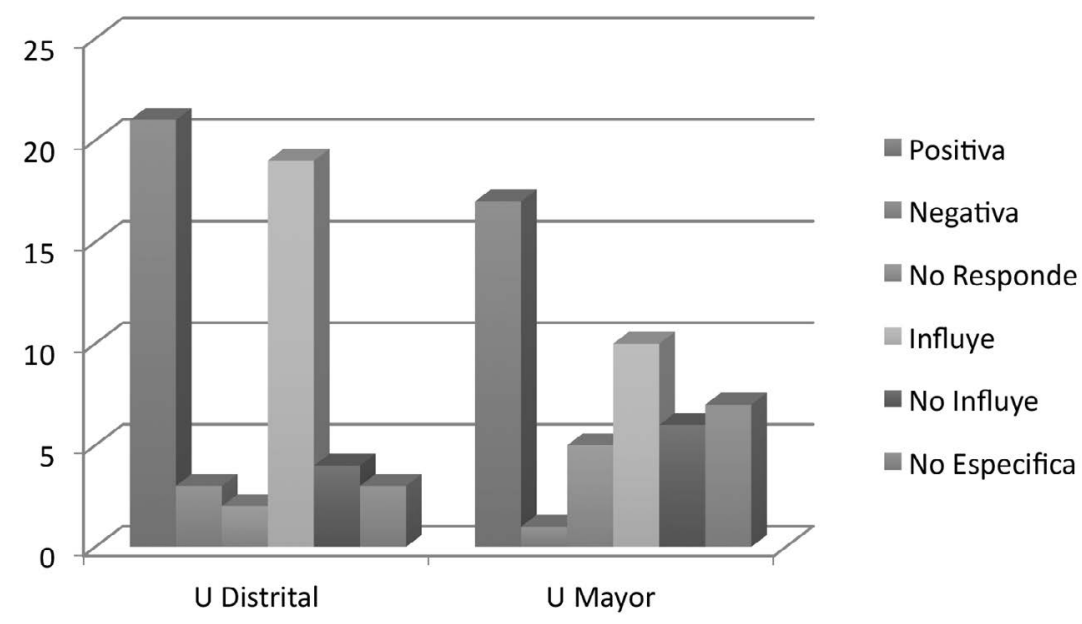

p. Figura deseo por ser docentes al finalizar la carrera.

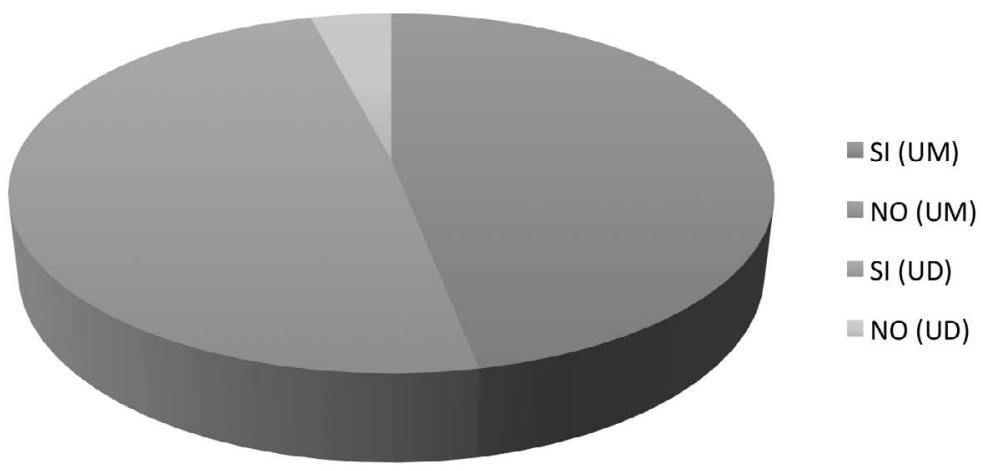

